\title{
SEXUALIDADE E AIDS NA TERCEIRA IDADE: ABORDAGEM NA CONSULTA MÉDICA
}

\author{
SEXUALITY AND AIDS IN OLD AGE: AN APPROACH IN MEDICAL CONSULTATION
}

\author{
Mariana Alves Santos ${ }^{\mathrm{a}}$, Barbara Santos Pires ${ }^{\mathrm{b}}$, Felipe Hoher Nahumc, Guilherme Antônio de \\ Paula Machadod, Gabriela Tomaz Silva ${ }^{\mathrm{e}}$, Gabriela Gonçalves Bangoim ${ }^{f}$, Ivone Panhoca $^{\mathrm{g}}$ \\ amari_88_454@hotmail.com, bbarbarapires_3@hotmail.com, cflp.nahum@hotmail.com, dguilhermeapm123@hotmail.com, \\ egabrielatomaz16@gmail.com, fgabibangoim@hotmail.com, si.panhoca@terra.com.br \\ Faculdade de Medicina da Universidade de Mogi das Cruzes - São Paulo (SP), Brasil
}

\section{RESUMO}

Objetivo: Investigar se na consulta médica há abordagem de assuntos sobre a sexualidade dos idosos por parte dos médicos e do próprio idoso e se é feito o pedido de exame para detectar o vírus HIV nessa população. Metodologia: Médicos das especialidades de geriatria, infectologia, urologia, clínica médica, cirurgia geral, ginecologia e psiquiatria de um hospital escola de Mogi das Cruzes (SP), totalizando 35 profissionais, responderam um questionário sobre aspectos de sexualidade abordados na consulta com o idoso. Resultados: A maioria dos médicos relatou que os idosos questionam, principalmente, a respeito de disfunção erétil, medicamento para ereção peniana e reposição hormonal. Entre os médicos, a maioria costuma abordar tais assuntos apenas quando há alguma queixa, sendo que dos 35 médicos apenas 9 conversam sobre o HIV e 13 sobre DST. A solicitação do exame de HIV não faz parte da rotina da maioria dos médicos, e a maioria dos idosos não pede para realizar o exame. Conclusão: Observou-se que há pouco diálogo entre a população idosa e os médicos sobre os temas DST/HIV e método contraceptivo. Inclusive, o exame para detectar o vírus não é realizado com frequência. Dessa forma, percebe-se a necessidade de inserir práticas assistenciais com o intuito de promover, prevenir e rastrear a infecção pelo vírus HIV na terceira idade.

Palavras-chave: Idoso; sexualidade; HIV; aids.

\section{ABSTRACT}

Objective: To investigate whether physicians or elderly patients address issues of sexuality during medical consultation and if tests are being done to detect the human immunodeficiency virus (HIV). Methodology: Doctors of geriatrics, infectious diseases, urology, internal medicine, general surgery, gynecology and psychiatry at a teaching hospital in Mogi das Cruzes (São Paulo), totaling 35 professionals, answered a questionnaire about sexuality issues addressed in consultation with the elderly. Results: Most doctors reported that the elderly have questioned mainly about erectile dysfunction, drugs for penile erection and hormone replacement and they only address such matters when there is a complaint. Only 9 out of 35 doctors talk about HIV, and 13 about sexually transmitted diseases (STD). The HIV testing request is not part of the routine of most doctors, and most seniors do not ask for the exam. Conclusion: It was observed that there is not enough dialogue between this population and physicians about STD/ HIV and contraception. Also, the exam to detect the virus is not taken very often. Thus, we see the need to integrate care practices in order to promote, prevent and track HIV infection in old age.

Keywords: Elderly; sexuality; HIV; AIDS. 


\section{Introdução}

Um dos grandes estereótipos sobre a terceira idade - no Brasil, adultos acima de 60 anos segundo a Organização Mundial de Saúde (OMS) - é relativo à sexualidade ${ }^{1}$. Os idosos tornaram-se mais ativos sexualmente após o desenvolvimento de medicamentos e próteses para disfunção erétil (em homens) e reposição hormonal (para as mulheres). Esses avanços tiveram como objetivo promover a qualidade de vida na terceira idade. Porém, a prevenção das doenças sexualmente transmissíveis (DSTs) não caminhou no mesmo passo ${ }^{2}$.

Nesse contexto, observa-se que o aumento no número de casos da síndrome da imunodeficiência adquirida (aids) é altamente considerável entre adultos mais velhos $^{3}$. Segundo o Boletim epidemiológico de aids e DST, a mortalidade por aids, nos últimos 10 anos, está diminuindo entre os mais jovens e aumentando nos idosos. Nos últimos 12 anos, a taxa de detecção do vírus HIV por 100 mil habitantes no Brasil, entre o público com mais de 60 anos, cresceu mais de $80 \%{ }^{3}$. A incidência de aids na população acima de 50 anos passou de $7,5 \% \mathrm{em}$ 1996 para $15,7 \%$ em 2006, ou seja, mais que dobrou em dez anos. Pensando nisso, em 2008, a campanha do Dia Mundial de Luta Contra a Aids teve como público-alvo a populaçáo heterossexual acima de 50 anos $^{4}$.

A infecção por HIV nos idosos, até os anos 1980, tinha como principal causa a transmissão sanguínea, mas atualmente é o contato sexual, principalmente entre homens homossexuais, com aumento dos casos também em heterossexuais ${ }^{5}$. Essa população tem vida sexual ativa, sendo que estudo realizado em 2008 mostrou que $73,1 \%$ havia feito sexo no último ano e apenas $22,3 \%$ havia usado preservativo na última relação ${ }^{4}$.

Segundo Souza et al. ${ }^{6}$, entre os idosos de 60-69 anos, $76,9 \%$ apresentavam vida sexual ativa, sendo $46,2 \%$ com parceiro fixo e $30,7 \%$ com múltiplos parceiros. No estudo realizado por Santos et al. ${ }^{7}, 44 \%$ dos idosos haviam tido ralação sexual com mais de um parceiro. A multiplicidade de parceiros se revela também na adesão dos idosos na contrataçáo de serviço sexual por profissionais do sexo, sendo que mais da metade dos idosos participantes do estudo adquiriu a infecção do HIV por meio desses profissionais ${ }^{7}$.

Tendo em vista que a aids é uma doença recente, da década de 1980, há dificuldade por parte dos idosos em perceber a necessidade do uso do preservativo ${ }^{8}$. Seu uso não é frequente nessa população, sendo seis vezes menor do que na população jovem, o que contribui para aumentar o número de casos de DST entre a terceira idade ${ }^{2}$.

Muitos são os fatores que elevam o número de idosos infectados; entre eles: a utilização de medicamentos para controlar a impotência sexual, o preconceito em relação à sexualidade na terceira idade, a falta de programas de saúde pública que informem os idosos sobre as formas de prevenção da doença e o baixo conhecimento dessa população a seu respeito?.

Além desses fatores, o paciente idoso frequentemente encontra-se em imunodepressáo, em que se observa manifestação de doenças oportunistas ${ }^{10}$. Assim, o idoso é diagnosticado com HIV, geralmente, após todos os outros possíveis diagnósticos serem descartados. Esse fato aponta para a importância de diferenciar as condiçóes de saúde que podem estar relacionadas à idade do idoso daquelas proporcionadas pelo vírus e as que podem estar presentes em ambas as situaçóes. Nesta última condição, encontra-se um amplo espectro de possíveis doenças ${ }^{11}$.

Pacientes idosos com HIV podem apresentar caquexia, astenia, anemia, diarreia, manifestaçôes fúngicas, fadiga, falta de ar, dor crônica, perda de peso, anorexia, alterações de memória, depressão, erupção cutânea e sintomas de aterosclerose ${ }^{11,12}$. Esses sintomas podem ser relacionados pelos profissionais da saúde apenas ao processo de envelhecimento, iniciando o tratamento sem identificar a presença do vírus. Além disso, dificuldades de memória recente e intelecto diminuído, que podem ser considerados como sintomas da doença de Alzheimer, também podem estar relacionados à infecçáo pelo HIV ${ }^{11}$.

Diante dessa problemática e da escassez de estudos que tratem da abordagem de profissionais médicos sobre a sexualidade com pacientes idosos, faz-se necessária a preocupação sobre esse tema, para que ações possam ser promovidas. Assim, este estudo se propôs a investigar se na consulta médica há abordagem de assuntos sobre a sexualidade dos idosos por parte dos médicos e do próprio idoso e se é feito o pedido de exame para detectar o vírus HIV nessa população.

\section{Metodologia}

Trata-se de um estudo transversal, de natureza quantitativa, cujos dados foram coletados em 2014/2015 em um hospital-escola onde o atendimento é $100 \%$ vinculado ao Sistema Único de Saúde (SUS), localizado no município de Mogi das Cruzes (SP).

O universo do estudo foi representado por médicos que trabalhavam no hospital supracitado, convidados a participar da pesquisa mediante preenchimento de um questionário elaborado pelos próprios acadêmicos e orientador, com 12 perguntas objetivas e 4 perguntas dissertativas a respeito dos assuntos sobre a sexualidade abordados em uma consulta com o paciente idoso. Em cada questão objetiva, o participante teve o direito de assinalar mais de uma resposta. Havia perguntas em relação ao questionamento por parte dos idosos e outras em relação ao questionamento por parte dos próprios 
médicos, e o único critério para participar do estudo foi a prática de atendimento e assistência a pacientes idosos. Sendo assim, apenas algumas especialidades foram selecionadas, sendo elas: clínica médica, cirurgia geral, ginecologia e obstetrícia, psiquiatria, urologia, geriatria e infectologia, totalizando 35 médicos.

Após a coleta, todos os dados foram processados e tabulados usando o programa BioEstat versão 5.3 e expressos em frequências simples, percentual e em gráficos de barras.

O presente trabalho foi aprovado pelo Comitê de Ética e Pesquisa, cujo número do parecer é 926.066. Todos os participantes assinaram o Termo de Consentimento Livre e Esclarecido (TCLE).

\section{Resultados}

Dentre os médicos entrevistados, a maioria relatou que os idosos os questionam a respeito da sexualidade durante a consulta médica (Tabela 1).

Tabela 1: Interesse dos idosos sobre a sexualidade segundo relato médico.

\begin{tabular}{ccc}
$\begin{array}{c}\text { Interesse dos idosos sobre a } \\
\text { sexualidade }\end{array}$ & Médicos (N) & Médicos (\%) \\
\hline Sim & 25 & 71,5 \\
Não & 10 & 28,5 \\
\hline
\end{tabular}

Fonte: Elaboração própria, com dados submetidos em Mogi das Cruzes (SP) em 2014/2015.

Pode-se observar na Tabela 2 que os temas mais questionados pelos idosos relacionados à sexualidade são disfunção erétil $(65,7 \%)$ e medicamentos para ereção $(45,7 \%)$. Os menos frequentes são reposição hormonal $(25,7 \%)$, DSTs em geral (excluindo HIV) $(22,8 \%)$; HIV propriamente dito $(11,4 \%)$ e métodos de prevenção $(2,8 \%)$.

Tabela 2: Assuntos de interesse dos idosos segundo relato médico.

\begin{tabular}{lcc} 
Assuntos de interesse dos idosos & $\begin{array}{c}\text { Médicos } \\
(\mathbf{N})\end{array}$ & $\begin{array}{c}\text { Médicos } \\
(\%)\end{array}$ \\
\hline Disfunção erétil & 23 & 65,7 \\
Medicamentos para ereção & 16 & 45,7 \\
Reposição hormonal & 9 & 25,7 \\
\hline DST & 8 & 22,8 \\
HIV & 4 & 11,4 \\
\hline Métodos de prevençáo & 1 & 2,8 \\
\hline
\end{tabular}

Fonte: Elaboração própria, com dados submetidos em Mogi das Cruzes (SP) em 2014/2015.

$\mathrm{Na}$ Tabela 3, encontram-se os temas mais frequentes questionados pelos médicos na consulta com o idoso.
Observa-se que $42,8 \%$ dos médicos só perguntam esses aspectos se houver alguma queixa por parte do idoso; $37,1 \%$, sobre DSTs em geral (excluindo HIV); 34,3\%, sobre reposição hormonal; 28,6\%, dispareunia; 25,7\%, medicamentos para ereção e HIV propriamente dito; e 22,8\% perguntam sobre métodos de prevenção.

Tabela 3: Assuntos abordados pelos médicos durante consulta.

\begin{tabular}{lcc} 
Assuntos abordados pelos médicos & $\begin{array}{c}\text { Médicos } \\
(\mathbf{N})\end{array}$ & $\begin{array}{c}\text { Médicos } \\
(\%)\end{array}$ \\
\hline Só pergunto se tiver queixa & 15 & 42,8 \\
\hline DST & 13 & 37,1 \\
\hline Reposição hormonal & 12 & 34,3 \\
Dispareunia & 10 & 28,6 \\
Medicamentos para ereção & 9 & 25,7 \\
HIV & 9 & 25,7 \\
Métodos de prevenção & 8 & 22,8
\end{tabular}

Fonte: Elaboraçâo própria, com dados submetidos em Mogi das Cruzes (SP) em 2014/2015.

Observa-se na Tabela 4 que a maior parte dos médicos não pede exame de sorologia para HIV para seus pacientes idosos se náo apresentarem alguma queixa, e a maioria dos idosos não pede exames para detectar o vírus, porém eles os solicitam mais do que os médicos (Tabela 5).

Tabela 4: Solicitação de sorologia para HIV em consulta de rotina.

\begin{tabular}{ccc}
$\begin{array}{c}\text { Solicitaçáo de sorologia para } \\
\text { HIV em consulta de rotina }\end{array}$ & Médicos (N) & Médicos (\%) \\
\hline Sim & 6 & 17,1 \\
Não & 28 & 80 \\
\hline
\end{tabular}

Fonte: Elaboração própria, com dados submetidos em Mogi das Cruzes (SP) em 2014/2015

Tabela 5: Solicitação de sorologia para HIV por parte dos pacientes idosos.

\begin{tabular}{cccc}
$\begin{array}{c}\text { Solicitaçáo de sorologia para } \\
\text { HIV por parte dos idosos }\end{array}$ & $\begin{array}{c}\text { Médicos } \\
(\mathbf{N})\end{array}$ & Médicos (\%) \\
Sim & 12 & 34,3 \\
\hline Não & 23 & 65,7 \\
\hline
\end{tabular}

Fonte: Elaboração própria, com dados submetidos em Mogi das Cruzes (SP) em 2014/2015.

As principais causas apontadas para os médicos solicitarem o exame de HIV para os idosos sáo: promiscuidade e infecção oportunista em primeiro lugar; infecçôes recorrentes, adenopatias, emagrecimento, DST e suspeita de imunodepressão em segundo lugar; relação sexual sem proteção, vida sexual ativa, queixas neurológicas, câncer de colo uterino, pré-operatório, rotina, contato com DST, relacionamento extraconjugal, câncer de pênis, condições do paciente e síndrome consumptiva em terceiro lugar. 


\section{Discussão}

Observou-se que a maior parte dos especialistas não pede o exame de sorologia para HIV para seus pacientes idosos se eles não apresentarem alguma queixa; e que a maioria dos idosos não pede o exame. Dessa forma, a assistência dada à terceira idade é voltada apenas para a livre demanda quando há a apresentação de queixas ${ }^{8}$.

Porém, a Secretaria de Estado da Saúde de São Paulo - segundo o Documento de diretrizes para prevenção das DST/aids em idosos ${ }^{11}$ - informa que, entre as atividades que podem ser desenvolvidas para as pessoas idosas, estão a solicitação do teste para as sorologias de hepatites B e C, sífilis e HIV, com a finalidade do diagnóstico precoce. Além disso, estimula a oferta de métodos contraceptivos, como o preservativo masculino e feminino, assim como o gel lubrificante, em unidades básicas de saúde e nos espaços de convivência dos idosos ${ }^{11}$.

O estudo realizado por Alencar e Ciosak ${ }^{13}$ verificou que, nos locais voltados à atenção primária, procurados pelos idosos, os profissionais não solicitavam a sorologia anti-HIV. Dessa forma, o diagnóstico acontece muitas vezes no nível secundário ou terciário, descobrindo tardiamente o vírus durante internaçáo hospitalar ou quando os idosos são atendidos em prontos-socorros. A importância do diagnóstico precoce também se apresenta, haja vista que o uso do preservativo náo é comum nessa população, e muitos idosos só iniciam o uso após o vírus ser diagnosticado.

O fato de o diálogo sobre a sexualidade nos atendimentos ocorrer apenas após o diagnóstico traz aos idosos o sentimento de serem assexuados frente à visão dos profissionais de saúde, pois as pessoas mais jovens são diagnosticadas com HIV mais precocemente ${ }^{14}$.

Os resultados mostraram que a maioria dos idosos pergunta aos médicos principalmente sobre disfunção erétil e medicamentos para impotência sexual. Além disso, mesmo havendo indícios de atividade sexual, o médico não pergunta na mesma proporção sobre DST/HIV e métodos de prevenção, uma vez que se observou que $37,1 \%$ dos médicos questionam sobre DST; $22,8 \%$, sobre métodos de prevenção; e $25,7 \%$, sobre HIV, sendo que $65,7 \%$ dos idosos perguntam sobre disfunção erétil e 45,7\% sobre medicamentos para ereçáo. Tais dados indicam a dificuldade que profissionais da saúde têm em abordar a questão da sexualidade com a população idosa ${ }^{13}$.

A literatura da área aponta que os profissionais da saúde se encontram despreparados, pois acreditam que os idosos não possuem vida sexual ativa. Dessa forma, descartam a possibilidade de o paciente estar infectado, o que dificulta o diagnóstico e leva a uma terapêutica incorreta, que tende a agravar o quadro, além de tornar o idoso um transmissor da doença?.

Assuntos como as DSTs, o HIV e os métodos de prevenção são menos consultados por parte dos idosos. Sendo assim, o paciente idoso também náo acredita que possa estar infectado ou não quer pensar nessa hipótese, formando-se, dessa forma, um ciclo vicioso, em que o médico não questiona e o paciente não aborda essa questấo ${ }^{15}$. Os familiares também, muitas vezes, não acreditam que nessa fase a pessoa tenha vida sexual ativa, e as formas de prevenção não são discutidas nem em casa nem nas unidades de saúde ${ }^{16}$. Assim, essa faixa etária não é priorizada pela saúde pública, e as campanhas sobre sexualidade, as doenças sexualmente transmissíveis e o uso de preservativos são apenas direcionadas ao público jovem e adulto "jovem".

Uma das estratégias necessárias para prevenir a infecção pelo vírus HIV é, além do uso de preservativos, aumentar a quantidade de indivíduos que realizam o exame para detectar o HIV, já que muitos casos de infecção ocorrem porque os transmissores não sabem que estão infectados ${ }^{17}$. Estudos apontam que maioria dos idosos nunca fez esse teste ${ }^{8,18,19}$, o que corrobora este estudo, visto que a maioria dos médicos não solicita o exame e a maioria dos idosos também não.

Outros estudos apontam que somente após a investigação de todas as possibilidades de doenças é que se solicita o exame para detectar o vírus HIV ${ }^{20,21}$. A precocidade do diagnóstico está relacionada com a visáo do profissional de saúde acerca da sexualidade na terceira idade ${ }^{22}$. Quando se tem este olhar, os profissionais solicitam o exame de HIV e investigam outras DSTs com mais naturalidade, como é percebido em outras faixas etárias ${ }^{22}$.

\section{Conclusão}

O presente trabalho nos mostra que há pouco diálogo entre idosos e médicos sobre as DSTs/HIV, os métodos contraceptivos e a realização do exame de HIV, apesar do interesse por parte dos idosos em disfunção erétil e medicamentos para a impotência sexual. Dessa forma, apesar do aumento do número de HIV/aids na terceira idade, pode-se pensar que talvez esse número esteja subestimado, uma vez que a prevenção e o rastreio para detectar o vírus não se apresentam frequentes nessa população.

Assim, percebe-se a necessidade de inserir práticas assistenciais com o intuito de promover, prevenir e rastrear a infecção pelo vírus HIV na terceira idade. É importante que tais assuntos sejam abordados pelos profissionais de saúde quando em contato com paciente idoso. Pode-se aproveitar o espaço que o idoso abre ao questionar sobre disfunção erétil, medicamentos para impotência sexual e reposição hormonal para investigar outros aspectos relacionados à sexualidade.

$\mathrm{O}$ presente estudo não teve a pretensão de esgotar todas as possibilidades do tema em questão, mas sim de ser uma contribuição a mais aos estudos da área, considerada a relevância do tema no atual momento. 
Espera-se que novos estudos surjam, aprofundando o tema e utilizando os resultados obtidos aqui.

\section{Referências}

1. Moraes KM, Vasconcelos DP, Silva ASR, Silva RCC, Santiago LMM, Freitas CASL. Companheirismo e sexualidade de casais na melhor idade: cuidando do casal idoso. Rev Bras Geriatr Gerontol. 2011;14(4):787-98.

2. Maschio MBM, Balbino AP, Souza PFR, Kalinke LP. Sexualidade na terceira idade: medidas de prevençáo para doenças sexualmente transmissíveis e aids. Rev Gaúcha Enferm. 2011 set [citado em 2016 jan 15];32(3):583-9. Disponível em: https://goo.gl/VUrDru

3. Ministério da Saúde. Boletim Epidemiológico de aids/DST. Brasília, DF: Ministério da Saúde; 2013.

4. Ministério da Saúde. Secretaria de Atençáo à Saúde. Departamento de DST, aids e hepatites virais. Brasília, DF: Ministério da Saúde; 2008 [citado em 2014 maio 20]. Disponível em: http://www.aids.gov.br/ campanhas/2008/38269>

5. Ministério da Saúde. Boletim Epidemiológico de DST/aids. Brasília, DF: Ministério da Saúde; 2010.

6. Souza LPS, Oliveira MVR, Silveira WRM, Figueiredo MFS, Messias RB, Silva JR. Análise da clientela idosa portadora de HIV atendida em um centro ambulatorial em Montes Claros, Minas Gerais. Rev Bras Geriatr Gerontol. 2012;15(4):767-76.

7. Santos NJS, Tayra A, Silva SR, Buchalla CM, Laurenti R. A aids no estado de São Paulo: as mudanças no perfil da epidemia e perspectivas da vigilância epidemiológica. Rev bras epidemiol [internet]. $2002 \mathrm{dez}$ [citado em 2016 jan 18];5(3):286-310. Disponível em: https://goo.gl/4YTO5E

8. Olivi M, Santana RG, Matias TAF. Comportamento, conhecimento e percepção de risco sobre doenças sexualmente transmissíveis em grupo de pessoas com 50 anos e mais de idade. Rev Latino-Am Enfermagem [internet]. 2008 ago [citado em 2016 jan 15];16(4):679-85. Disponível em: https://goo.gl/N3gOJt

9. Batista AFO, Marques APO, Leal MCC, Marino JG, Melo HMA. Idosos: associação entre o conhecimento da aids, atividade sexual e condiçôes sociodemográficas. Rev Bras Geriatr Gerontol. 2011;14(1):39-48.

10. Toledo LSG, Maciel ELN, Rodrigues LCM, Sá RT, Fregona G. Características e tendência da aids entre idosos no estado do Espírito Santo. Rev Soc Bras Med Trop. 2010;43(3):264-7.

11. Secretaria da Saúde do Estado de São Paulo. Centro de Referência e Treinamento em DST/aids. Documento de diretrizes para prevenção das DST/aids em idosos. Bepa. 2011;8(92):15-23.

12. Silva HR, Marreiros MOC, Figueiredo TS, Figueiredo, MLF. Características clínico-epidemiológicas de pacientes idosos com aids em hospital de referência, Teresina-PI, 1996 a 2009. Epidemiol Serv Saúde. 2011;20(4):499-507.

13. Alencar RA, Ciosak SI. O diagnóstico tardio e as vulnerabilidades dos idosos vivendo com HIV/aids. Rev Esc Enferm USP [internet]. 2015 abr [citado em 2016 jan 17];49(2):229-35. Disponível em: https://goo.gl/Xrlkha

14. Ellman TM, Sexton ME, Warshafsky D, Sobieszczyk ME, Morrison EA. A forgotten population: older adults with newly diagnosed HIV. AIDS Patient Care STDs. 2014;28(10):530-6.

15. Brasileiro M, Freitas MIF. Representaçōes sociais sobre aids de pessoas acima de 50 anos de idade, infectadas pelo HIV. Rev Latino-Am Enfermagem [internet]. 2006 out [citado em 2016 jan 18];14(5):789-95. Disponível em: https://goo.gl/b89t2G

16. Araújo VLB, De Brito DMS, Gimeniz MT, Queiroz TA, Tavares CM. Características da aids na terceira idade em um hospital de referência do estado do Ceará, Brasil. Rev Bras Epidemiol. 2007 dez [citado em 2016 jan 18];10(4):54454. Disponível em: https://goo.gl/mOQdAx

17. Jansen RS. HIV/aids in persons 50 years of age and older. Atlanta: Center for Infectious Diseases Center for Diseases Control and Prevention. 2005 [citado em 2014 abr 15]. Disponível em: https://goo.gl/HPDUKM

18. Lazzarotto AR, Kramer AS, Hädrich M, Tonin M, Caputo P, Sprinz E. O conhecimento de HIV/aids na terceira idade: estudo epidemiológico no Vale do Sinos, Rio Grande do Sul, Brasil. Ciênc Saúde Coletiva [internet]. 2008 dez [citado em 2016 jan 18];13(6):1833-40. Disponível em: https:// goo.gl/RAoG3u

19. Melo HMA, Leal MCC, Marques APO, Marino JG. O conhecimento sobre aids de homens idosos e adultos jovens: um estudo sobre a percepção desta doença. Ciênc Saúde Coletiva. 2012;17(1):43-53.

20. Linley L, Prejean J, An Q, Chen M, Hall HI. Racial/ethnic disparities in HIV diagnoses among persons aged 50 years and older in 37 US States, 2005-2008. Am J Public Health [internet]. 2012 ago [citado em 2016 jan 18];102(8):152734. Disponível em: https://goo.gl/GmEZhw

21. Slinkard MS, Kazer MW. Older adults and HIV and STI screening: the patient perspective. Geriatr Nurs. 2011;32(5):341-9.

22. Alencar RA, Ciosak SI. Early diagnosis of HIV in the elderly population: a brief review of the literature. Online Braz J Nurs. 2010 ago [citado em 2016 jan 18];9(2):1-10. Disponível em: https://goo.gl/OOVJnF

Como citar este artigo:

Santos MA, Pires BS, Nahum FH, Machado GAP, Silva GT, Bangoim GG, Panhoca I. Sexualidade e aids na terceira idade: abordagem na consulta médica. Rev. Aten. Saúde. 2017;15(51):18-22. 